\title{
ESTUDIO DE LA INTERCULTURALIDAD COMO COMPETENCIA TRANSVERSAL EN LAS AULAS DE CIENCIAS DE LA COMUNICACIÓN
}

\section{STUDY OF INTERCULTURAL AND CROSS-CURRICULAR CLASSROOM OF COMMUNICATION SCIENCES}

Francisco Cabezuelo Lorenzo: Prof. Doctor Facultad de Humanidades y Comunicación. Universidad San Pablo - CEU. Madrid (España) francisco.cabezuelolorenzo@ceu.es

María José Pérez Serrano: Prof. Doctora Facultad de Ciencias de la Comunicación. Universidad de San Jorge. Zaragoza (España)

mjperez@usj.es

\section{CURRÍCULUM VITAE}

Doctor Europeo en 'Tecnologías, Estructuras y Tratamientos de la Información' por la Facultad de Ciencias de la Información de la Universidad Complutense de Madrid. Cuenta con diferentes postgrados universitarios realizados en diversas universidades europeas y norteamericanas. Es investigador activo del Grupo Europeo de Investigación MEDIADEM (Séptimo Programa Marco de I+D la Comisión Europea) a través de la Universidad de Castilla-La Mancha, del Grupo de Investigación en Formación Universitaria en Comunicación (GIFUCOM) en el Espacio Europeo de Educación Superior (EEES) de la Universitat Abat Oliba CEU de Barcelona y del Grupo de Investigación en EduComunicación (GIEC). Ha sido becario de postgrado de la Fundación "La Caixa" en las prestigiosas McGill University y Concordia 
University (Canadá) y de la Fundación Caja Madrid en The Queen's University of Belfast (Irlanda del Norte, Reino Unido). Igualmente, fue becario IES-UCM en la University of Saint Thomas en Minneapolis-Saint Paul (Estados Unidos). En su trayectoria profesional destacan numerosos puestos de responsabilidad como la dirección ejecutiva del Pabellón de la Santa Sede en la Exposición Internacional de Zaragoza 2008. Como periodista ha trabajado varios años en diferentes secciones de la agencia de noticias Europa Press, además de en diferentes emisoras de radio de ámbito estatal y en publicaciones de tirada nacional. En el campo de la comunicación institucional y las relaciones públicas ha desarrollado diferentes tareas en la Oficina del Parlamento Europeo en España y en el Departamento de Prensa del Senado. Ha sido profesor en la Facultad de Ciencias Humanas y de la Comunicación de la Universidad Europea Miguel de Cervantes de Valladolid (España) y en la Facultad de Ciencias de la Comunicación de la Universidad San Jorge de Zaragoza (España). En la actualidad es profesor de la Teoría de la Comunicación y de la Información en la Universidad CEU San Pablo en Madrid (España).

Licenciada en Periodismo, doctora en Ciencias de la Información por la Universidad Complutense de Madrid y MBA en la especialidad de Gestión Financiera. Su tesis doctoral ha sido merecedora del Premio Extraordinario de Doctorado del curso académico 2006-2007. Está acreditada por la ANECA como Profesor Ayudante Doctor, Profesor de Universidad Privada y Profesor Contratado Doctor. Ha pertenecido, entre otros, al cuerpo docente de las facultades de Comunicación de la Universidad San Jorge y de la Universidad Pontificia de Salamanca y del Instituto de Postgrado y Formación Continua de la Universidad Pontificia de Comillas (ICADE), así como del Departamento de Periodismo IV (Empresa Informativa) de la Facultad de Ciencias de la Información de la Universidad Complutense de Madrid, donde en estos momentos imparte docencia. Su trayectoria y producción científica se vincula 
al estudio de la Empresa Informativa y, específicamente, a la concentración de medios de comunicación y su influencia en el pluralismo. Es autora de comunicaciones y artículos; ha desarrollado estancias académicas en centros universitarios nacionales e internacionales, y forma parte de grupos de investigación

\section{RESUMEN}

En general, la interculturalidad se presenta como un nuevo reto en las aulas. En este sentido, las universitarias tampoco son una excepción y menos aún las que se dedican a enseñar Ciencias de la Comunicación. La interculturalidad debe ser entendida como un principio, pero también como un proyecto para la mejora de las acciones y relaciones entre las culturas. En el plano didáctico, se configura mediante el diálogo y el encuentro compartido entre todos los estudiantes, el profesorado y las comunidades participantes. La interculturalidad debe buscar el encuentro creativo de experiencias humanas diversas. Además, necesita nuevas concepciones y prácticas educativas centradas en la equidad, la solidaridad y el acercamiento plural entre todas las personas y pueblos. En el nuevo Espacio Europeo de Educación Superior se sitúa como una de las competencias transversales más importantes.

\section{PALABRAS CLAVE}

Interculturalidad - Comunicación - Competencia - Didáctica - Espacio Europeo de Educación Superior

\section{ABSTRACT}

The phenomenon of interculturality appears as a new challenge in the classrooms. It is also a great challenge in the field of the Communication Sciences and the Mass 
Media Studies. Interculturality must be defined as the principle and project for the improvement of the actions and relations among cultures, by means of dialog and meetings shared between all the students, the teaching staff and the communities which decide to take part in the process. Interculturality must seek for the creative meeting of human diverse experiences. In addition, it needs new conceptions and educational practices centred on the equity, the solidarity and the plural approximation among all the persons and peoples. It is a key pile in the new European Space of Higher Education.

\section{KEY WORDS}

Intercultural - Communication - Competence - Methods of teaching - European Higher Education Area

\section{ÍNDICE}

1. Introducción y justificación del tema

2. Claves epistemológicas y propuestas metodológicas

3. Planteamiento teórico

3.1. La interculturalidad: un fenómeno creciente

3.2. Elementos de la nueva docencia universitaria: didáctica y competencias 4. La "metáfora del iceberg" y la Comunicación

4.1. Planteamiento

4.2. Aplicaciones

5. Conclusiones

6. Bibliografía y fuentes 


\section{TEXTO}

\section{Introducción y justificación del tema}

La interculturalidad se ha convertido en una realidad que no puede ser ignorada. Esta realidad ha llegado a las aulas de centros de primaria y secundaria pero también a las de las facultades y centros de postgrado vinculados a las Ciencias de la Información y la Comunicación. Pero, ¿qué es la interculturalidad? ¿cuenta el docente con las herramientas necesarias para interiorizarla en su ritmo didáctico? La interculturalidad es "el principio y proyecto para la mejora de las acciones y relaciones entre las culturas, configurada mediante el diálogo y el encuentro compartido entre todos los estudiantes, el profesorado y las comunidades participantes" (Domínguez, 2006: 12); o, dicho de otro modo, "la síntesis superadora y vivencial del ser y saber humano que se reconoce a sí mismo en la relación y el encuentro creativo de experiencias humanas diversas, que necesita nuevas concepciones y prácticas educativas centradas en la equidad, la solidaridad y el acercamiento plural entre todas las personas y pueblos, con el máximo respeto a la identidad en el horizonte de nuevas actuaciones de carácter global" (Medina et al.,2004).

Este planteamiento de apertura y entendimiento se incardina perfectamente en la interdependencia que exige el mundo en el que vivimos que, sea en el contexto que sea, motiva la colaboración entre ciudadanos de países antes aislados. Como profesores universitarios, hemos vivido esta realidad gracias a la implantación de forma experimental y avanzada de nuestros estudios conforme al Espacio Europeo de Educación Superior (EEES) y a diferentes experiencias de intercambio académico (de alumnos, profesores y personal no docente) gracias al ya asentado programa Erasmus. Esta concepción intercultural ha llegado a la universidad y, aunque no sin 
dificultades, se está traduciendo en estrategias que persiguen la consecución de objetivos como la formación de ciudadanos libres a través de un sistema educativo de calidad, principio que se sitúa como uno de los valores positivos con los que se identifica nuestra civilización.

Partiendo de la Declaración de Bolonia entendida como un hito temporal y funcional para el cambio y también como intersección evolutiva que tiene como fin la imprescindible necesidad de cambiar los napoleónicos basamentos universitarios, se detectan nuevos retos para la universidad europea del siglo XXI. Este nuevo modelo busca revivir aquello de lo que su nomenclatura hacía gala: un modelo universal con una trascendental repercusión de las metodologías de enseñanza-aprendizaje. Lo que se hacía patente a través de algunos de los objetivos marcados en 1999: "la promoción de la cooperación europea para asegurar un nivel de calidad para el desarrollo de criterios y metodologías comparables"; "la promoción de una necesaria dimensión europea en la educación superior con particular énfasis en el desarrollo curricular", y "la promoción de la movilidad y remoción de obstáculos para el ejercicio libre de la misma por los estudiantes, profesores y personal administrativo de las universidades y otras instituciones de enseñanza superior europea"1

Pero, si se pudiera establecer un paralelismo entre todos estos elementos de análisis y vincularlo a un proceso complejo de management, tras los objetivos vendrían los cambios, como resultados de una necesidad constante de adaptación a las circunstancias que impone el entorno. Y, con este nuevo panorama, a la universidad, se nos exige aprender a enseñar de otra manera. Dicho de otra forma, "los tiempos que corren están exigiendo de quienes nos dedicamos a la formación de profesionales un estado de permanente alerta, de reconceptualización e indagación de nuevas y viejas herramientas, aquellas que tengan en cuenta las exigencias de los nuevos contextos de enseñanza y aprendizaje caracterizados por la integración de las nuevas 
tecnologías y, sobre todo, por el ineludible protagonismo de sus participantes" (Agra, et. al: 2008).

De esta idea sobresalen dos elementos fundamentales: de un lado, el contexto de enseñanza-aprendizaje, que ha cambiado debido, sobre todo, a la nueva realidad social que representa el microcosmos del aula, donde la diversidad es una clara y desde nuestro punto de vista- positiva característica, y, de otro, el protagonismo creciente de los discentes, que aportan, además, de un background esencial de conocimiento, un equipaje imprescindible de experiencia vital, que enriquece y aporta al resto, convirtiendo al docente en plausible testigo de los cambios, conductor ineludible de las nuevas circunstancias, y "exprimidor" de lo mejor de cada una de las realidades presentes.

La interculturalidad es, ante todo, un "reto socio-educativo y profesional" (Domínguez, 2006: 11) y, en el contexto de la European Higher Education Area (EHEA) vinculado a los estudios de Comunicación, se sitúa como una competencia transversal en la nueva formación de nuestros futuros profesionales. En esta línea, este trabajo persigue el análisis de diversas experiencias de nuevos planteamientos docentes derivados de Bolonia teniendo como eje conductor la competencia de la interculturalidad y, como centro gravitatorio, al alumno.

1-V. http://www.crueuta.info/web_crue/docs/declaracion_bolonia.pdf. 


\section{Claves epistemológicas y propuestas metodológicas}

La incursión de la interculturalidad en la realidad y práctica docentes es un campo muy tratado y motiva profusamente la preocupación constante de profesores, pedagogos e investigadores. Lo que no es tan habitual es que se vincule a la docencia universitaria y, menos aún, a la especificidad de los estudios de Comunicación. Conscientes de que este trabajo representa un acercamiento; de que la incursión en un campo debe ser, siempre, tibia y cautelosa, y que, ante la comunidad científica, se exige, en todo caso, una imprescindible captatio benevolentia, señalamos, en primer lugar, los límites desde los que se procede. Aunque, fundamentado en experiencias reales, el prisma desde el que se escribe no es de la especialización pedagógica y, por lo tanto, este trabajo representa una aproximación, una incursión con todas las cautelas, sin mayor pretensión que el fin para el que se ha creado.

La proposición conjetural que sirve de punto de partida es que la realidad intercultural en que vivimos y el EEES implican una transformación en los planteamientos didácticos y exige un esfuerzo de adecuación pedagógica y de planificación de la que antes estaba alejada la docencia universitaria. Asimismo, el objetivo marcado se completa con la exposición, en forma de aplicación práctica, de la "metáfora del iceberg" dentro de las aulas de segundo ciclo de las Licenciaturas en Periodismo y Publicidad y Relaciones Públicas de dos universidades españolas durante el curso 2008/2009.

Este trabajo ha de ser entendido metodológicamente a partir de las definiciones de interculturalidad, dada en las primeras líneas de este trabajo, de competencia y de didáctica, como la reconstrucción de una disciplina poniéndose en lugar del alumno, todo ello inserto en un proceder discursivo marcado por la deducción. Partiendo de esa base y con los mimbres citados (además, de la ejemplificación de la "metáfora del 
iceberg") para llevar a cabo el proceder discursivo se aplicará el método científico; se incidirá en el hipotético-deductivo para su claridad y rigor intelectual; se utilizará el análisis cualitativo, y se completará con el análisis de las variables que sustentan la investigación y sus conclusiones.

\section{Planteamiento teórico}

En la actualidad, el concepto de interculturalidad aparece vinculado, de forma casi exclusiva, al de diversidad cultural, pero, muchas veces, se echa de menos dar un paso más y darse cuenta de que se trata, a través del diálogo y el respeto mutuo, de posibilitar el encuentro entre culturas, sin olvidar el contexto social e ideológico preexistente. Teniendo este telón de fondo y sabiendo que las disquisiciones de tipo teórico y semántico conducen a una reducción del espectro real y, de algún modo, limitan y constriñen esa realidad, resulta imprescindible fundamentar teóricamente el "iter" explicativo para no incurrir en errores cognitivos de base.

\subsection{La interculturalidad: un fenómeno creciente}

Aunque durante muchos años, España poseía una realidad diferente respecto a otros países de su entorno (como Francia, Alemania, Bélgica, Inglaterra), poco a poco, nuestra realidad socio-demográfica ha dejado de ser peculiar. La inmigración empezó a crecer a en los años noventa con fuerza y se ha incrementado de manera exponencial en la última década y media, hasta llegar a situarse, dentro del espectro docente, como un reto creciente. Así, ahora, es más que evidente la presencia de alumnos de diferentes nacionalidades en las aulas españolas, que motiva -en algunas ocasiones- la aparición de un abanico de situaciones complejas planteadas por los discentes que, en algunas ocasiones, tienen que ver con una plausible carencia idiomática e, incluso, una deficiente escolarización previa. 
Evidentemente, estos acontecimientos son menos frecuentes en las aulas universitarias que en las propias de las etapas de formación obligatoria. Pero dado que el EEES no significa, solamente, la puesta en marcha de un lugar de encuentro común y unas reglas básicas de entendimiento para las universidades europeas, sino que implica una transformación radical en los planteamientos didácticos y la consecución de unos objetivos (calidad, movilidad, diversidad y competitividad) que conduzcan al alumno hacia una formación integral y pegada a la realidad circundante, tampoco podemos olvidar los profesores universitarios cuál es el mapa cultural que tenemos delante y cómo se producen las relaciones de integración entre distintos grupos sociales a los que damos clase.

Diferentes estudios sobre interculturalidad y multiculturalidad marcan que, la primera se refiere a la interacción y comunicación entre diferentes culturas, de una forma respetuosa, horizontal y sinérgica, donde no se concibe que un grupo cultural pueda estar sobredimensionado con respecto a otro y donde se favorece, en todo momento, la integración y convivencia de ambas partes. En las relaciones interculturales se establece un vínculo basado en el respeto a la diversidad y el enriquecimiento mutuo, algo que es vital en estos tiempos, y más si cabe en estos de crisis y conflictos. La formación en cuestiones interculturales enseña que los conflictos se resuelven a través del respeto, el diálogo, la escucha mutua, la concertación y la unión de buenas voluntades.

Pero por bien definida no significa que sea bien aplicada y entendida, ya que es un reto para quien enseña adoptar y adaptar esa interculturalidad y ello exige la “capacitación del profesorado para la creación de climas y procesos interculturales ha de fundamentarse en la reflexión meticulosa acerca del nivel de identidad con la tarea educativa y el equilibrio socio-emocional sin el cual es difícil entender los retos de las nuevas culturas, valores y expectativas de todas las personas, singularmente 
de los estudiantes y familias" (Domínguez, 2006: 15). En resumen, y desde el punto de vista del docente, la actual situación en la que nos encontramos exige dar un paso más en la formación del profesor para aprender a enseñar en el contexto intercultural.

\subsection{Elementos de la nueva docencia universitaria: didáctica y competencias}

En el sistema educativo no universitario, la Ley 10/2002, de Calidad de la Educación (LOCE), de 23 de diciembre, hacía hincapié en la diversidad del alumnado, en su tratamiento y en la oferta de distintos itinerarios que se acomoden a las posibilidades del mismo. Pero donde esta norma "es absolutamente innovadora es en el nuevo tratamiento que da al fenómeno de la inmigración, buscando preparar adultos que se integren totalmente en los niveles social y económico y en preocuparse por reforzar significativamente el sistema de oportunidades para todo el alumnado" (Perulles; Banciella, 2003: 3-4).

En línea semejante, pero desde distinto prisma ideológico, la vigente Ley 2/2006, de Educación (LOE), de 3 de mayo, en su Preámbulo resalta la importancia de la educación de los jóvenes y considera que es el medio más adecuado "para construir su personalidad, desarrollar al máximo sus capacidades, conformar su propia identidad personal y configurar su comprensión de la realidad, integrando la dimensión cognoscitiva, la afectiva y la axiológica", así como "para garantizar el ejercicio de la ciudadanía democrática, fomentar la convivencia, el respeto a las diferencias individuales, promover la solidaridad y evitar la discriminación".

El la Integración del Sistema Universitario Español en el Espacio Europeo de Educación Superior (MECD, 2003) expone que "los objetivos formativos de las enseñanzas oficiales de nivel de grado tendrán, con carácter general, una orientación 
profesional, es decir, deberán proporcionar una formación universitaria en la que se integren armónicamente las competencias genéricas básicas, las competencias transversales relacionadas con la formación integral de las personas y las competencias más específicas que posibiliten una orientación profesional que permita a los titulados una integración en el mercado de trabajo".

Teniendo en cuenta lo que ya ha sido comentado respecto al hecho de que la interculturalidad esté más estudiada e interiorizada en el sistema educativo previo a la Universidad, podemos decir que en el bloque segundo de competencias de las que habla el Documento-Marco es donde ésta se incluye, tal como ha sido explicada en el epígrafe anterior.

Con el fin de detallar qué se entiende por competencia, podemos señalar que para Lasnier (2000), es "un saber hacer complejo resultado de la integración, movilización y adecuación de capacidad y habilidades (de orden cognitivo, afectivo, psicomotor o social) y de conocimientos, utilizados eficazmente en situaciones que tenga un carácter común)".

Pero estas competencias o, dicho de una manera sintagmáticamente más correcta, al aprendizaje basado en competencias, aunque nuevo en el contexto de la Educación Superior, tiene una larga tradición. "La historia de la Educación Basada en Normas de Competencias(EBNC) se remonta a los años treinta del siglo XX en los Estados Unidos". Sin embargo, su manifestación más reciente data de hace más de quince años, como un interés más económico que educativo, con el fin de adecuar la educación y capacitación vocacionales a las necesidades de la industria. Desde entonces la EBNC ha sido un concepto muy controvertido entre representantes de los sectores industriales, gubernamentales y educativos, pero también ha generado consenso en torno a que es un buen punto de partida para elevar los niveles de competencias en un determinado país, para aumentar los recursos que se invierten 
en programas de capacitación y para hacer posible que otras instituciones no gubernamentales impartan capacitación (Gonczi, 2001: 19).

El concepto de competencia es diverso, según el ángulo del cual se mire o el énfasis que se le otorgue a uno u otro elemento, pero el más generalizado y aceptado es el de "saber hacer en un contexto". El "saber hacer", lejos de entenderse como "hacer" a secas, requiere de conocimiento (teórico, práctico o teórico-práctico), afectividad, compromiso, cooperación y cumplimiento, todo lo cual se expresa en el desempeño, también de tipo teórico, práctico o teórico-práctico (Posada, 2008).

Esta incorporación de nuevas exigencias académicas a la docencia universitaria (competencias, interculturalidad, etc.) han motivado un esfuerzo de reformulación de las áreas de especialización y la creación ex novo de guías docentes 2 y de planteamientos ad hoc. Es aquí donde adquiere importancia la definición de didáctica como la reconstrucción de una disciplina poniéndose en lugar de un alumno que ya no es $-\mathrm{y}$, prácticamente, nunca lo ha sido- estándar y uniforme, la creación de un material que facilite el aprendizaje autónomo y la definición de lo que Zabalza (2006) denomina "coreografía didáctica", donde los protagonistas del proceso (profesores, discentes e institución educativa) tienen que tomar conciencia de la nueva situación; transformar sus roles, tareas y procedimientos, y actuar en consecuencia.

Explicados ya los pilares teóricos sobre los que se asienta este trabajo, llega el momento de observar prácticamente cómo la competencia de la interculturalidad está presente en los estudios de Comunicación, a través de diversas experiencias, para ello se explicará, en primer lugar, la "metáfora del iceberg". 
2- Pauta "cuasi-contractual" del decurso académico diario y plasmación ponderadamente útil del "conjunto coherente de técnicas y acciones, lógicamente coordinadas, para dirigir el aprendizaje del discente hacia determinados objetivos" (Fernández, 2005) inserto en una política universitaria que lleva por bandera el progreso constante.

\section{La "metáfora del iceberg" y la Comunicación}

Sin duda alguna, una de analogías 3 más adecuadas para comprender el funcionamiento de la cultura es la "metáfora del iceberg". Ésta viene a decir que lo que sobresale del agua es equivalente a lo que notamos en la interacción diaria con otras personas. En este grupo de variables encontraríamos elementos como la apariencia física de las personas, la comida, bebida y alimentos, y hasta la propia lengua (idioma) de cada uno. Por otro lado, lo que se encuentra debajo del agua aquello que no se ve ni se palpa a simple vista- sería el grupo de variables formado por las creencias, valores y percepciones que tiene la gente sobre la vida desde su propia cultura. 


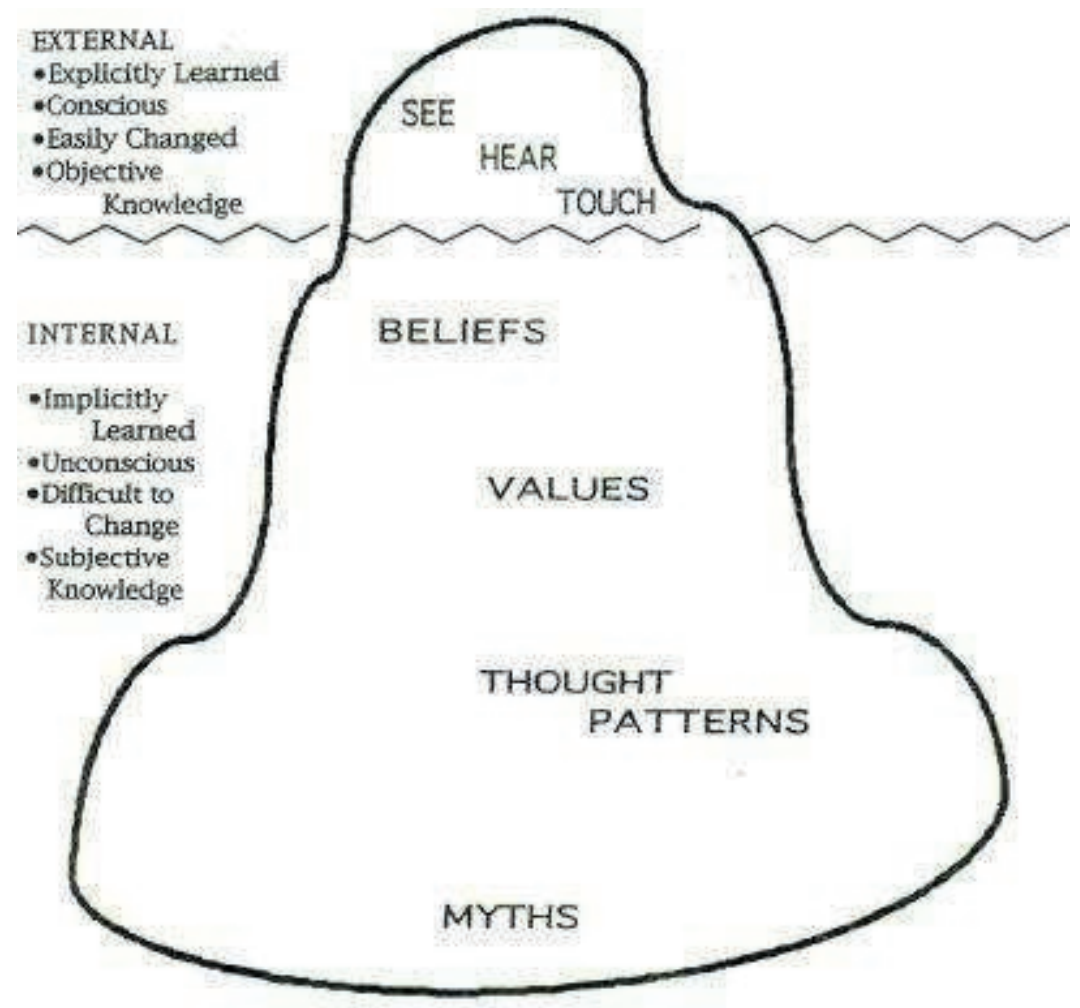

De este modo, la "metáfora del iceberg" viene a señalar grosso modo que las cosas que están más a la vista suponen una pequeñísima parte de lo que somos, y como lo más importante es lo que cuesta más descubrir y lo que hace que nuestro iceberg, nuestra vida, se mantenga en la superficie.

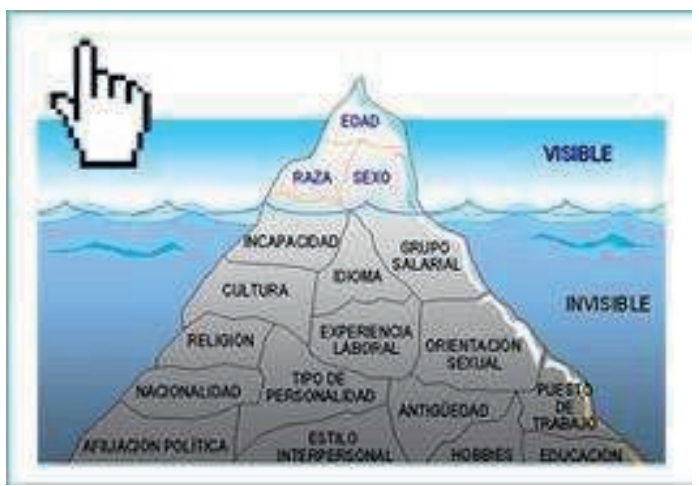

La realidad es que la cultura es trasmitida de generación en generación. Por tanto, estamos ante un comportamiento aprendido y un patrón oculto de relaciones 
humanas, expresiones y puntos de vista compartidos por un grupo determinado de personas. Los aspectos de la cultura que permanecen escondidos se llaman "protocolos". Son los códigos que a menudo crean dificultades interculturales porque tienen efectos significativos en nuestro comportamiento y en la forma en que interactuamos con los que nos rodean.

Las interacciones que definen una relación interpersonal pueden ser observables y explícitas, como sucede en el diálogo, o no verbales, como el lenguaje corporal. Cuando hablamos de relaciones interpersonales nos referimos a asociaciones sociales, interacciones y contactos entre dos o más personas, ya sea que se encuentren frente a frente, o se conozcan exclusivamente a través de las nuevas redes sociales online como Facebook, Tuenti, eLink, etc 4, o el correo electrónico y/o el chat (conversaciones en Internet).

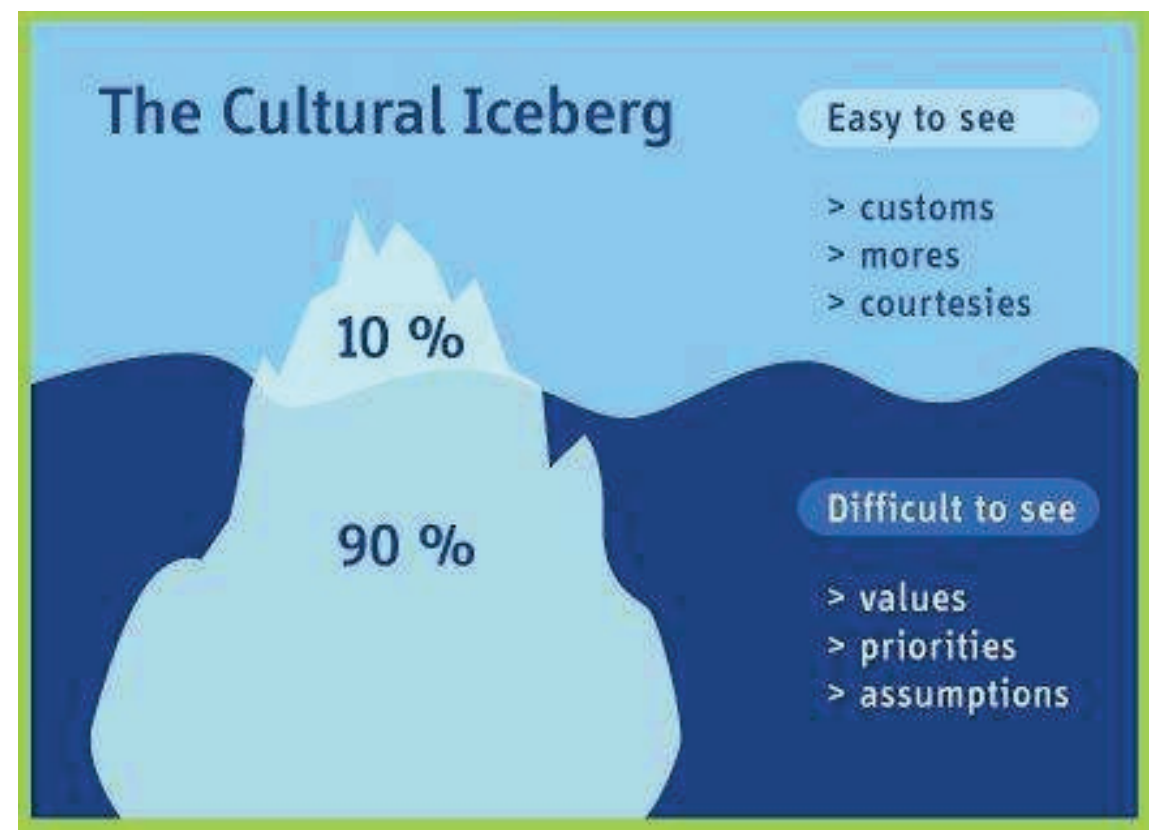

Muy ligado a esta metáfora está también la cuestión de la comunicación no verbal. Mediante el proceso de aprehensión de la misma, se adquieren competencias que se 
pueden mejorar interactuando con personas de diferentes culturas, lo que posibilita que las habilidades también se incrementen. Mediante la enseñanza de la comunicación no verbal, se puede alcanzar la adaptación y el éxito en medios distintos a los que estamos acostumbrados, ya sea nuestro propio entorno u otro medio diferente.

En este mundo globalizado, como este tipo de comunicación está estrechamente relacionada con la cultura, es esencial entender las claves que ofrece el nuevo medio con la mayor rapidez posible. Paradójicamente más del 85 \% de la comunicación de los seres humanos es de este tipo, es decir, no se manifiesta a través de las palabras. La función de la comunicación no verbal es complementar, regular, sustituir y resaltar la comunicación verbal. De este modo, todo el mundo necesita, para poder interpretar una nueva cultura diferente, algo tan necesario como el deseo de mantener una relación armoniosa con el otro, con el que es diferente. El éxito que ésta genere dependerá de que se establezca un diálogo abierto y franco entre las dos partes, basado en el respeto mutuo.

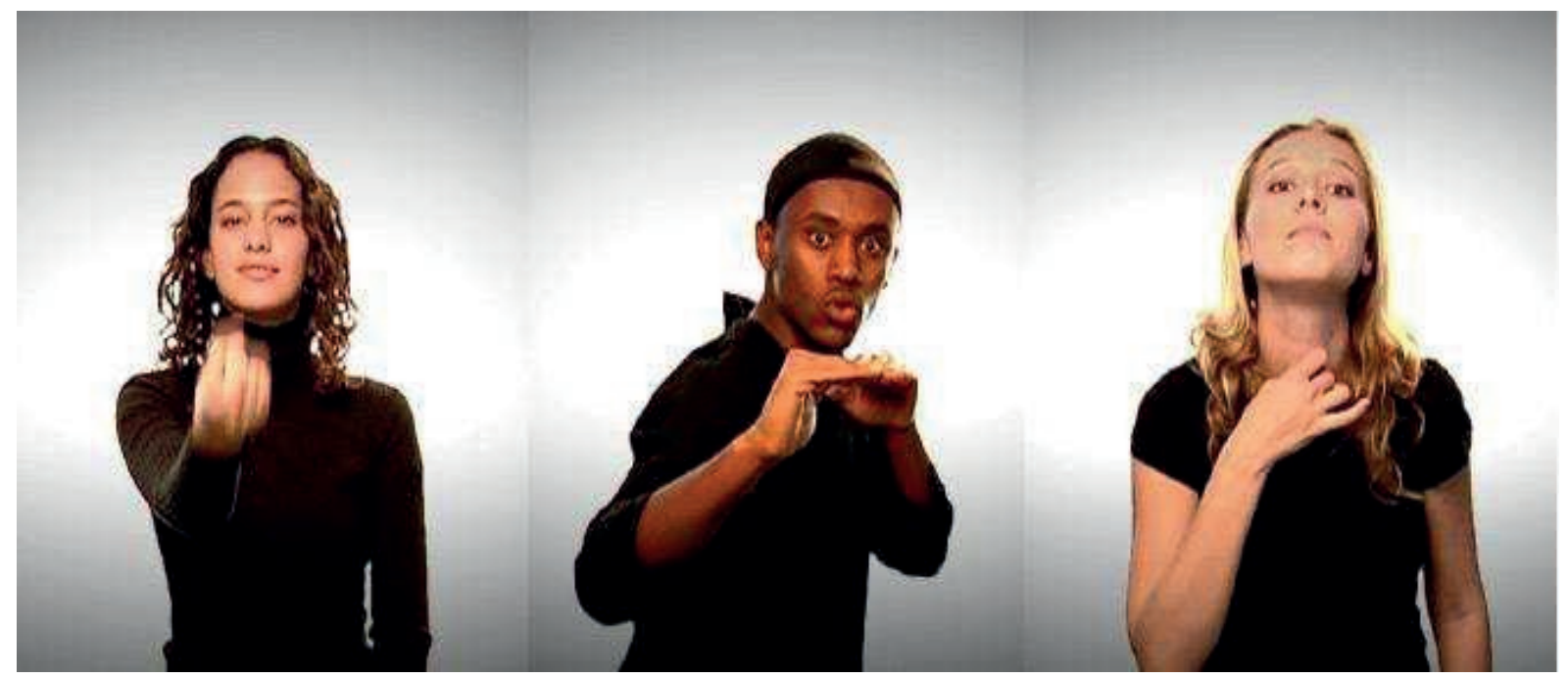


La comunicación no verbal es, como su propio nombre indica, un lenguaje sin palabras. Se basa en la kinésica, prosémica y cronémica 5. Abarca desde factores tan evidentes y externos como la forma de vestir y los gestos, hasta otros mucho más sutiles como la manera de movernos en el espacio, la distancia que mantenemos al interactuar con otros, el contacto físico y la percepción del tiempo. Algo tan cotidiano como un saludo entre dos personas conocidas, puede variar considerablemente de una cultura a otra. Para algunos no involucra ningún tipo de contacto físico, mientras que para otros puede requerir un beso en la mejilla, en algunos casos dos, en otros tres, y en el caso de los parisinos, cuatro. Este tipo de comunicación ocurre a nivel inconsciente, se manifiesta a través de diversos canales y se aprende por imitación (Pease, 2006).

3- Las imágenes que ilustran la metáfora del iceberg han sido tomadas del servidor gratuito Google Pictures.

4- Éstas son solo algunas de las posibilidades de redes sociales en internet.

5- Estudio de la comunicación a través del movimiento, el espacio, el tiempo y la proximidad, respectivamente.

\subsection{Planteamiento}

Tal y como hemos ido viendo en este trabajo, el mundo de la interculturalidad está estrechamente ligado al mundo de la comunicación. Comunicación no verbal e interculturalidad guardan fuertes lazos que las vinculan. Por este motivo, como profesores de universidad, proponemos algunas actividades o acciones que sirvan 
para el estudio de la interculturalidad en nuestras aulas, donde conviven alumnos de diferentes backgrounds, culturas, grupos étcnicos, idiomas nativos y clase social. De ahí la necesidad de llevar al aula actividades orientadas a desarrollar dicha consciencia y aprovechar las oportunidades que surjan para fomentar el diálogo en las aulas.

\subsection{Aplicaciones}

A continuación, se recogen una serie de actividades que fueron desarrolladas por nuestros alumnos, inspiradas en otros ejercicios propuestos por profesoras de español como lengua extranjera (García; Massó, 2006) y adaptadas al desarrollo de competencias para los futuros profesionales del Periodismo, la Publicidad y las Relaciones Públicas, que tienen como objetivo desarrollar estrategias vinculadas a la interculturalidad.

\section{a) Actividad 1}

En el contexto de estudio de las características de la globalización económica, se propuso un debate a los alumnos de segundo ciclo de las titulaciones de Comunicación sobre el fenómeno de la inmigración en nuestro país. Ésta sería la pregunta: ¿Qué deberían hacer los inmigrantes al llegar a tu país? Al término de la misma, y para que utilicen las herramientas de su futura profesión, se les propone la redacción de una pieza interpretativa.

\section{b) Actividad 2}

En esta segunda actividad, se les pidió a los alumnos, en un esfuerzo de revisión de sus conocimientos previos, que reflexionaran, a través de una pieza del género opinión, sobre la cultura del otro y cómo su carencia puede llevar a visiones estereotipadas 6. La pregunta a la que deberían responder es la siguiente: ¿Qué sabes 
de la familia española? Deberían responder a preguntas del tipo (entre otras): ¿Cuántos hijos tienen? ¿Cuánto presupuesto mensual manejan? ¿Qué porcentajes de divorcios hay? ¿A qué edad se emancipan los hijos? Buscábamos demostrar que desconocen la realidad propia y ajena.

\section{c) Actividad 3}

El objetivo de esta tercera actividad estuvo centrado en el desarrollo de la consciencia intercultural poniéndose en el lugar (papel) del otro. Tuvieron que responder a las siguientes preguntas: ¿Qué harías si fueras un estudiante internacional de intercambio en un país desconocido? Imagina que por cuestiones académicas te vas a estudiar Periodismo, Publicidad o Relaciones Públicas a otro país. Haz un análisis DAFO 7 sobre esta nueva realidad.

\section{d) Actividad 4}

En este caso se trataba de reflexionar sobre una cuestión de género y la "nouniversalidad" de los valores. Se propuso un debate sobre el rol de las mujeres en la publicidad en los diferentes países del mundo, haciendo hincapié en las diferencias entre Europa, Norteamérica, el Mundo Árabe, o Asia oriental.

\section{e) Actividad 5}

Y, por último, esta práctica propuso la creación de un autorretrato cultural con el fin de tomar conciencia del carácter dinámico de la cultura, puesto que una cultura cambia, evoluciona, y se transforma de generación en generación. Por ese motivo, se les encargó hacer un reportaje sobre tradiciones familiares, cambios a lo largo del tiempo, fiestas populares, historias de sus familias, y otros temas en los que se mostró los cambios sufridos y evolución fruto del paso del tiempo. Los alumnos investigaron durante una semana $y$, tras la redacción del trabajo, fue expuesto en 
clase, para incidir en la simbiosis (interculturalidad-comunicación) que antes hemos detallado.

6- Los estereotipos son ampliamente difundidos por los medios de comunicación, tanto por la prensa, la radio y la televisión. Igualmente, la publicidad se basa en estereotipos para sus fines.

7- Debilidades, Amenazas, Fortalezas y Oportunidades.

\section{Conclusiones}

Partiendo de que nuestra hipótesis ha sido corroborada, como apuntes conclusivos podemos reiterar los siguientes:

1. En la actualidad, la interculturalidad se ha convertido en una realidad que no puede ser ignorada. Esta realidad ha llegado a las aulas, por eso desde el campo académico se necesitan nuevas concepciones y prácticas educativas centradas en valores universales, con el máximo respeto a la pluralidad del actual mundo globalizado en el que vivimos.

2. La inclusión de la interculturalidad en la realidad y práctica docentes es un campo muy tratado y motiva profusamente la preocupación constante de profesores, pedagogos e investigadores. Lo que no es tan habitual es que se vincule a la docencia universitaria y, menos aún, a la especificidad de los estudios de las Facultades de Ciencias de la Comunicación. 
3. El Espacio Europeo de Educación Superior (EEES) implican una transformación en los planteamientos didácticos y exige un esfuerzo de adecuación pedagógica y de planificación de la que antes estaba alejada la docencia universitaria en la que esté presente la interculturalidad.

4. La "metáfora del iceberg" es un buen ejemplo que a modo de metáfora viene a decirnos que lo que sobresale del agua es equivalente a lo que notamos en la interacción diaria con otras personas, y lo que se encuentra debajo del agua -aquello que no se ve ni se palpa a simple vista- sería el grupo de variables formado por las creencias, valores y percepciones que tiene la gente sobre la vida desde su propia cultura.

5. Los futuros profesionales de la Comunicación que actualmente se forman como alumnos en las titulaciones de Periodismo, Publicidad y Relaciones Públicas necesitan una formación específica en valores universales que les capaciten para un mundo contemporáneo en el que cada vez tiene más sentido el término globalización.

6. La interculturalidad es una competencia que debe desarrollarse de forma transversal en diferentes materias de las impartidas en los estudios, grados y especializaciones de las Ciencias de la Comunicación.

\section{Bibliografía y fuentes}

AGRA, María Jesús; GEWERC, Adriana; MONTERO, Lourdes: “El portafolios como herramienta de análisis en experiencias de formación on-line y presenciales". En: II Congreso Europeo de Tecnologías de la Información en la Educación y en la Ciudadanía. Barcelona, 26, 27 y 28 de Junio de 2006, [en línea], 
<http://www.usal.es/ ofeees/NUEVAS_METODOLOGIAS/PORTAFOLIO/c45.p df> (28 abril 2009).

BANKS, James A.: Handbook of Research on Multicultural Education. San Francisco, 2004. Ed. Jossey-Bass.

DECLARACIÓN DE BOLONIA, 1999, [en línea], <http://www.crueuta.info/web_crue/docs/declaracion_bolonia.pdf>, (02 Jul. 2008).

DOMÍNGUEZ GARRIDO, $\mathrm{M}^{\mathrm{a}}$ Concepción: Investigación y formación del profesorado en una sociedad intercultural. Madrid, 2006. Ed. Universitas.

FERNÁNDEZ MARCH, Amparo: “Nuevas metodologías docentes”, Valencia, 2006. Universidad Politécnica de Valencia, línea], <http://www.upm.es/estudios/eduSup/actividades/Nuevas_metodologias_docen tes/NUEVAS_METODOLOGIAS_DOCENTES.pdf>, (28 febrero 2009).

GARCÍA-VIÑÓ, Mónica; MASSÓ PORCAR, Amparo: Propuestas para desarrollar la consciencia intercultural en el aula de español lengua. En: RedELE, no 7. Madrid, 2006.

GONCZI, Andrew: “Análisis de las tendencias internacionales y de los avances en educación y capacitación basadas en normas de competencias". En: ARGÜELLES, Antonio; GONCZI, Andrew: Educación y capacitación basada en normas de competencias: una perspectiva internacional. México, 2001. Ed. Limusa. 
LASNIER, François : Réussir la formation par competences. Montreal, 2006. Ed. Guérin.

Ley 10/2002, de Calidad de la Educación (LOCE), de 23 de diciembre.

Ley 2/2006, de Educación (LOE), de 3 de mayo.

MEDINA REVILLA, Antonio; RODRÍGUEZ MARCOS, Ana; IBÁÑEZ DE ALDECOA, Alicia (coords.): Interculturalidad: Formación del Profesorado y Educación. Madrid, 2005. Ed. Pearson Prentice Hall.

MINISTERIO DE EDUCACIÓN, CULTURA Y DEPORTE: “Documento-Marco sobre la integración del Sistema Universitario español en el Espacio Europeo de Enseñanza Superior". Madrid, 2003, [en línea], <http://webs.uvigo.es/esct/doc_marco_MECD_2003.pdf>, (26 de abril de 2009).

PAIGE, R. Michael (ed.): Education for the Intercultural Experience. Yarmouth, 1993. Intercultural Press, Inc.

PAIGE, R. Michael; COHEN, Andrew D; KAPPLER, Barbara; CHI, Julie C; LASSEGARD, James P.: Maximizing Study Abroad. A Student's Guide to Strategies for Language and Culture Learning and Use. Minnesota, 2002. Center for Advanced Research on Language Acquisition. Office of Internacional Programs. University of Minnesota.

PEASE, Allan: El lenguaje del cuerpo: cómo interpretar a los demás a través de sus gestos. Barcelona, 2006. Amat Editorial. 
PÉREZ SERRANO, María José; CABEZUELO LORENZO, Francisco: Las nuevas tendencias ciberperiodísticas en el marco del EEES. En: FLORES VIVAR, Jesús M.; ESTEVE RAMÍREZ, Francisco: Periodismo Web 2.0. Madrid, 2009. Editorial Fragua.

PÉREZ SERRANO, María José; FANJUL PEYRÓ, Carlos; CABEZUELO LORENZO, Francisco: "Planificación docente y por competencias en asignaturas adaptadas al EEES: el caso CREPEC". En: IV Congreso Internacional "La renovación de metodologías docentes centradas en el nuevo proceso de aprendizaje del alumno", Valladolid, 16 y 17 de septiembre de 2008.

PERULLES RULL, Antoni; BANCIELLA SUÁREZ, María Jesús: “La interculturalidad en las aulas: líneas de tratamiento", 2003, [en línea], $<$ http://www.forumeducacio.org/aportdebat/la_interculturalidad_en_las_aulas.pd f>, (27 de abril de 2009).

POSADA ÁLVAREZ, Rodolfo M., "Formación superior basada en competencias, interdisciplinariedad y trabajo autónomo del estudiante". En: Revista Iberoamericana de Educación, 2004, [en línea], http://www.rieoei.org/ deloslectores/648Posada.PDF, (28 febrero 2009).

SIERRA SÁNCHEZ, Javier; CABEZUELO LORENZO, Francisco: La formación en competencias digitales de los futuros periodistas: la experiencia curricular de la Universidad San Jorge. En PINTO, Ricardo Jorge; SOUSA, Jorge Pedro (coords): Os Jovens e a Renovação do Jornalismo. Porto, 2008. Edições Universidade Fernando Pessoa.

ZABALZA, Miguel Á.: Competencias docentes del profesorado universitario. Calidad y desarrollo profesional.Madrid, 2006. Ed. Narcea. 